Supporting Information

\title{
Understanding and Countering Illumination-Sensitive Dark Current: Toward Organic Photodetectors with Reliable High Detectivity
}

Jianfei Huang, ${ }^{a}$ Jaewon Lee, ${ }^{b}$ Hidenori Nakayama,${ }^{c}$ Max Schrock, ${ }^{a}$ David Xi Cao, ${ }^{a}$ Kilwon Cho, ${ }^{d}$

Guillermo C. Bazan, ${ }^{a}$ Thuc-Quyen Nguyen*, a

${ }^{a}$ Center for Polymers and Organic Solids, University of California, Santa Barbara, CA 93106, United

States

${ }^{\mathrm{b}}$ Department of Chemical Engineering and Applied Chemistry, Chungnam National University, Daejeon 34134, Republic of Korea

${ }^{\mathrm{c}}$ Organic Materials Laboratory, Science \& Innovation Center, Mitsubishi Chemical Corporation, 1000 Kamoshida-cho, Aoba-ku, Yokohama, Kanagawa 227-8502, Japan

${ }^{\mathrm{d}}$ Center for Advanced Soft Electronics, Department of Chemical Engineering, Pohang University of Science and Technology, Pohang, 37673, Republic of Korea 


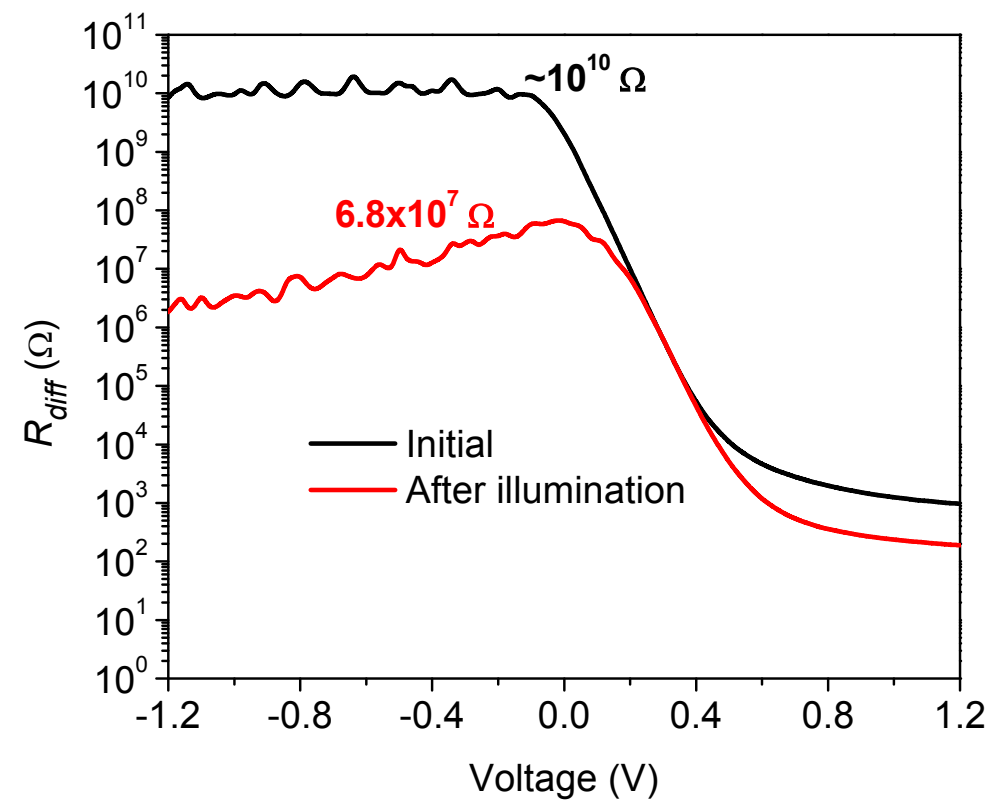

Figure S1. Differential resistance and estimated shunt resistance of the ZnO ETL based inverted OPDs before and after illumination.

The decrease of shunt resistance leads to increase of thermal noise according the equation:

$$
I_{t h}=\sqrt{\frac{4 k T B}{R_{s h}}}
$$

where $k$ is the Boltzmann constant, $T$ is the absolute temperature, $B$ is the bandwidth and $R_{s h}$ is the shunt resistance. The thermal noise before and after illumination is $1.3 \times 10^{-15} \mathrm{~A} \mathrm{~Hz}^{-1 / 2}$ and $1.5 \times 10^{-14} \mathrm{~A} \mathrm{~Hz}^{-1 / 2}$. Nevertheless, the typical shot noise goes from $5 \times 10^{-15} \mathrm{~A} \mathrm{~Hz}^{-1 / 2}$ to $2 \times 10^{-13} \mathrm{~A} \mathrm{~Hz}^{-1 / 2}$ after the illumination, manifesting a larger increase and a more dominant source of noise at the operating condition. 


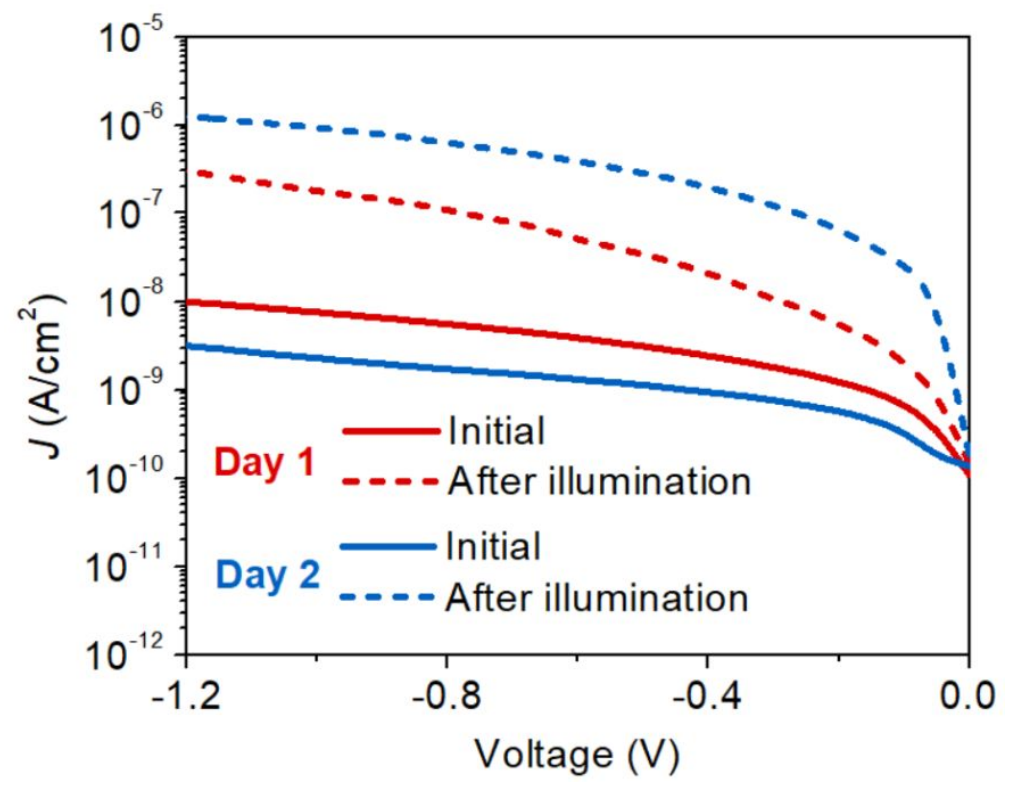

Figure S2. Dark $J-V$ curves before and after illumination, measured on the day of device fabrication (Day 1 , red) and the next day (Day 2, blue), respectively. 

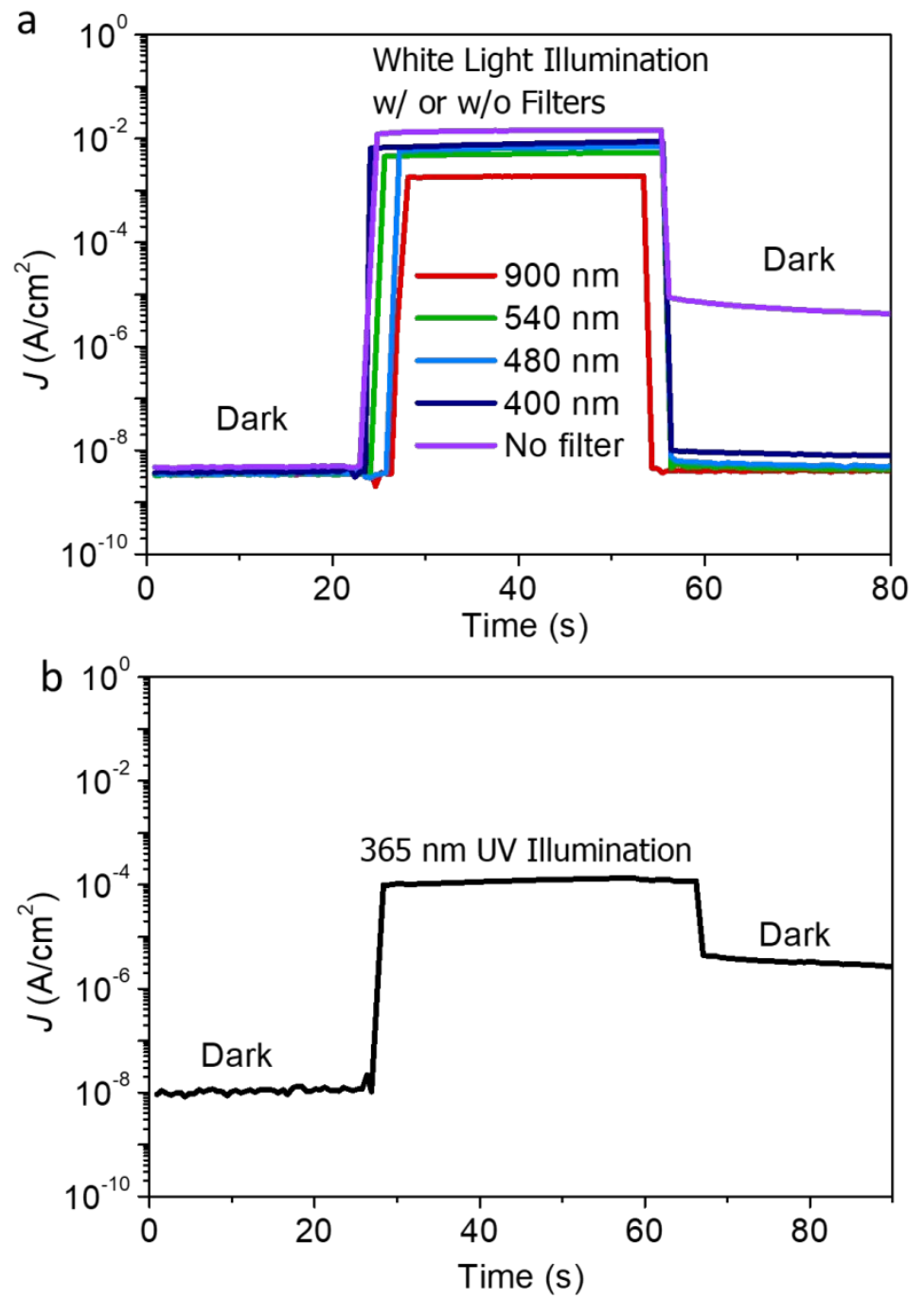

Figure S3. (a) Current density versus time of a freshly made OPD device measured at $-1 \mathrm{~V}$. The illumination processes have long-pass filtering that blocks the light with wavelength below the indicated numbers. The tests were done in the same order as shown in Figure 2a. (b) Current density versus time of a freshly made OPD device measured at $-1 \mathrm{~V}$ under the dark, UV illumination $\left(\sim 7 \mathrm{~mW} / \mathrm{cm}^{2}\right)$ and dark conditions. 


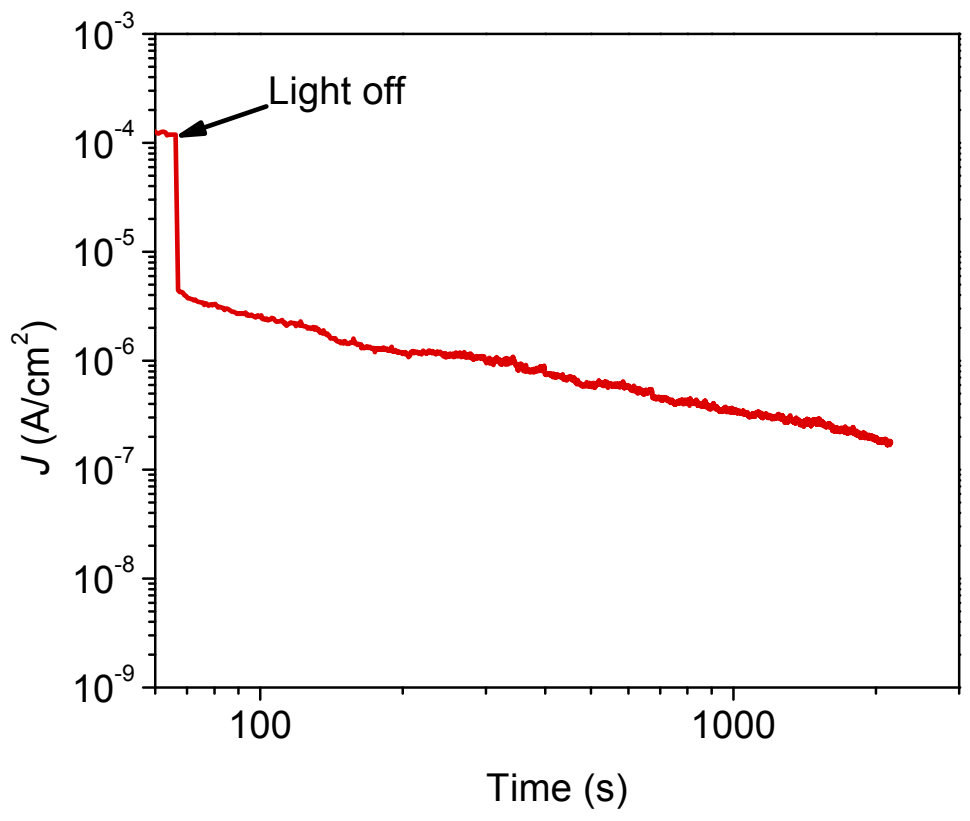

Figure S4. Current density versus time of a freshly made OPD device measured at $-1 \mathrm{~V}$ after UV illumination $\left(\sim 7 \mathrm{~mW} / \mathrm{cm}^{2}\right)$ is removed. 


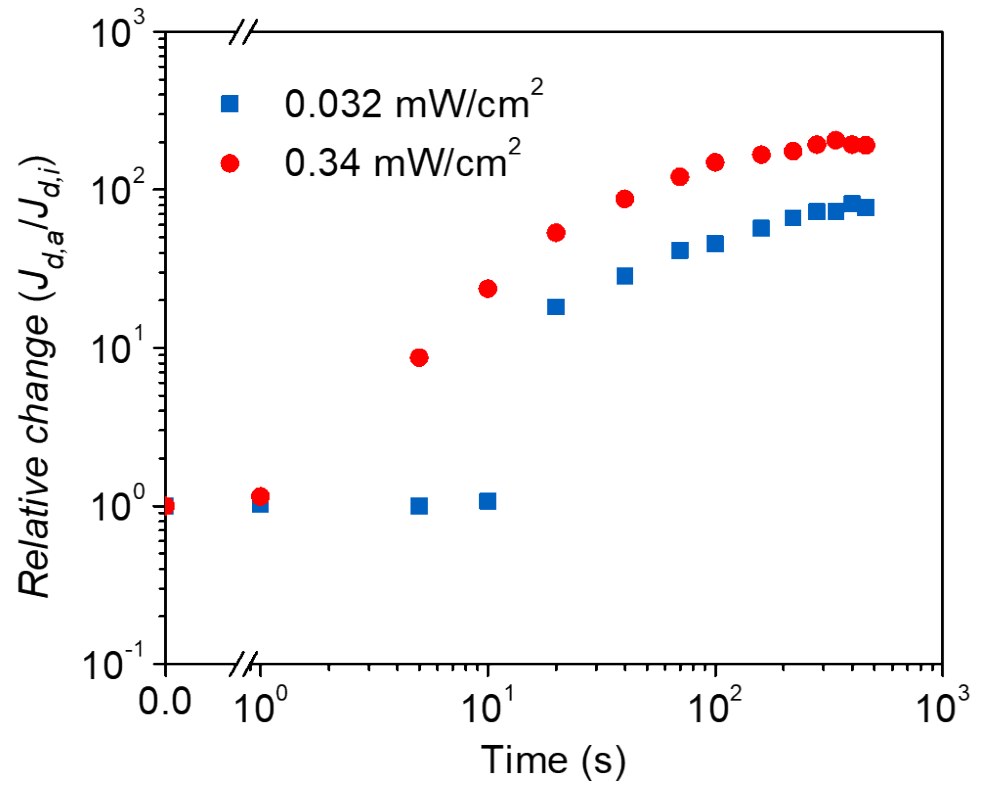

Figure S5. Relative change of dark current vs accumulated time of $365 \mathrm{~nm}$ UV light exposure. $J_{d, i}$ and $J_{d, a}$ is the initial and after-illumination dark current. 

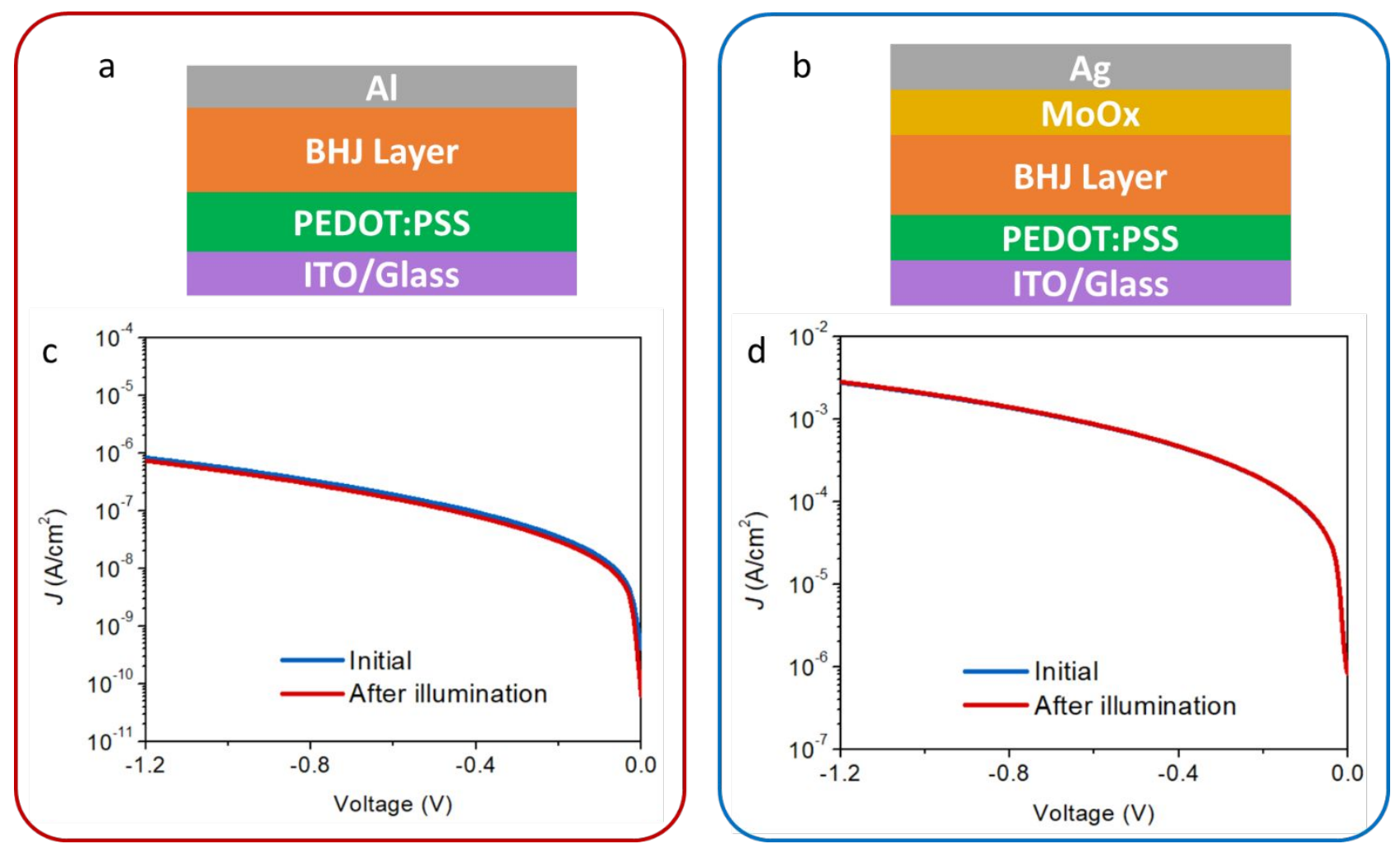

Figure S6. (a-b) Device structures and (c-d) dark $J-V$ curves of the control group devices. 

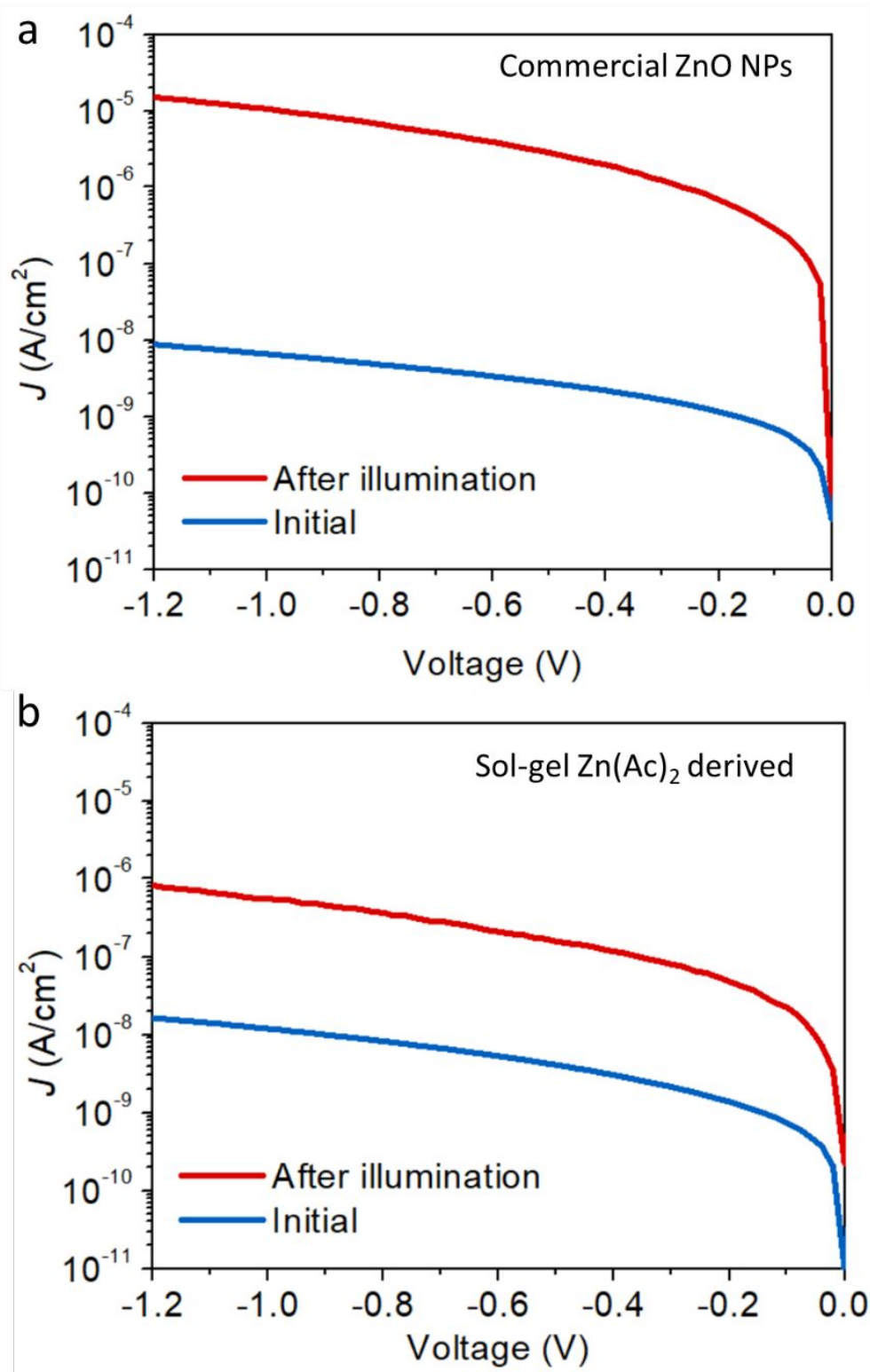

Figure S7. Dark $J-V$ characteristics of the inverted OPDs with ZnO interlayer prepared by different methods: (a) commercial ZnO nanoparticles (NPs) suspension and (b) zinc acetate-based sol-gel method. 


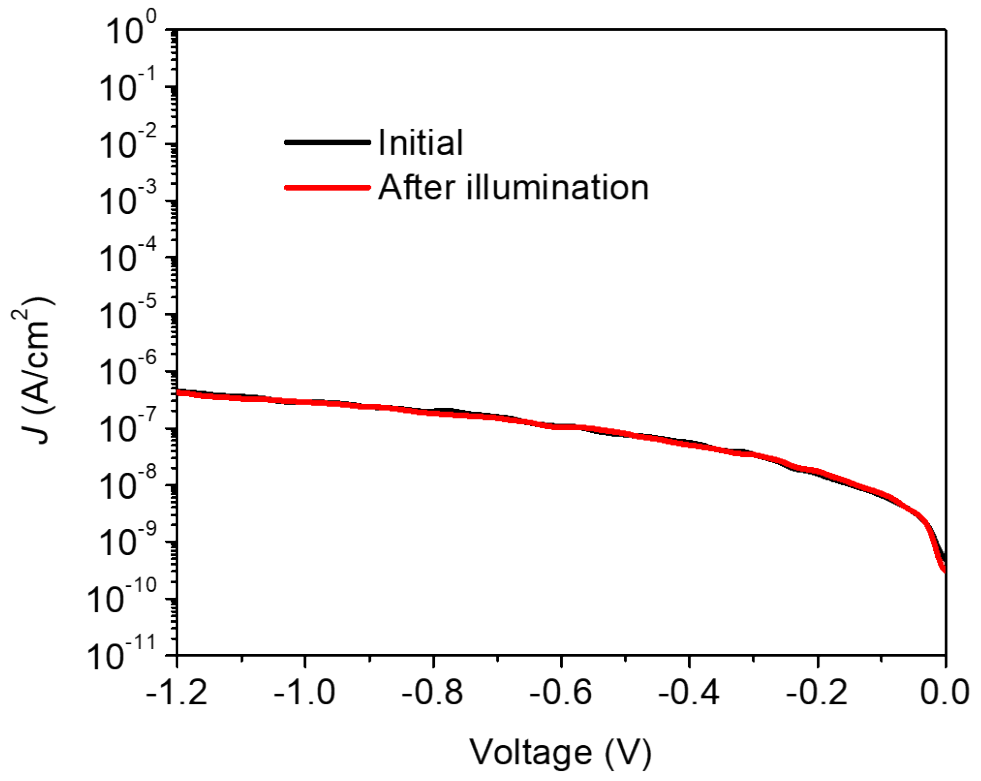

Figure S8. Dark $J-V$ curves of conventional structure device with ZnO ETL (TIO/PEDOT:PSS/Active Layer/ZnO/Al). 

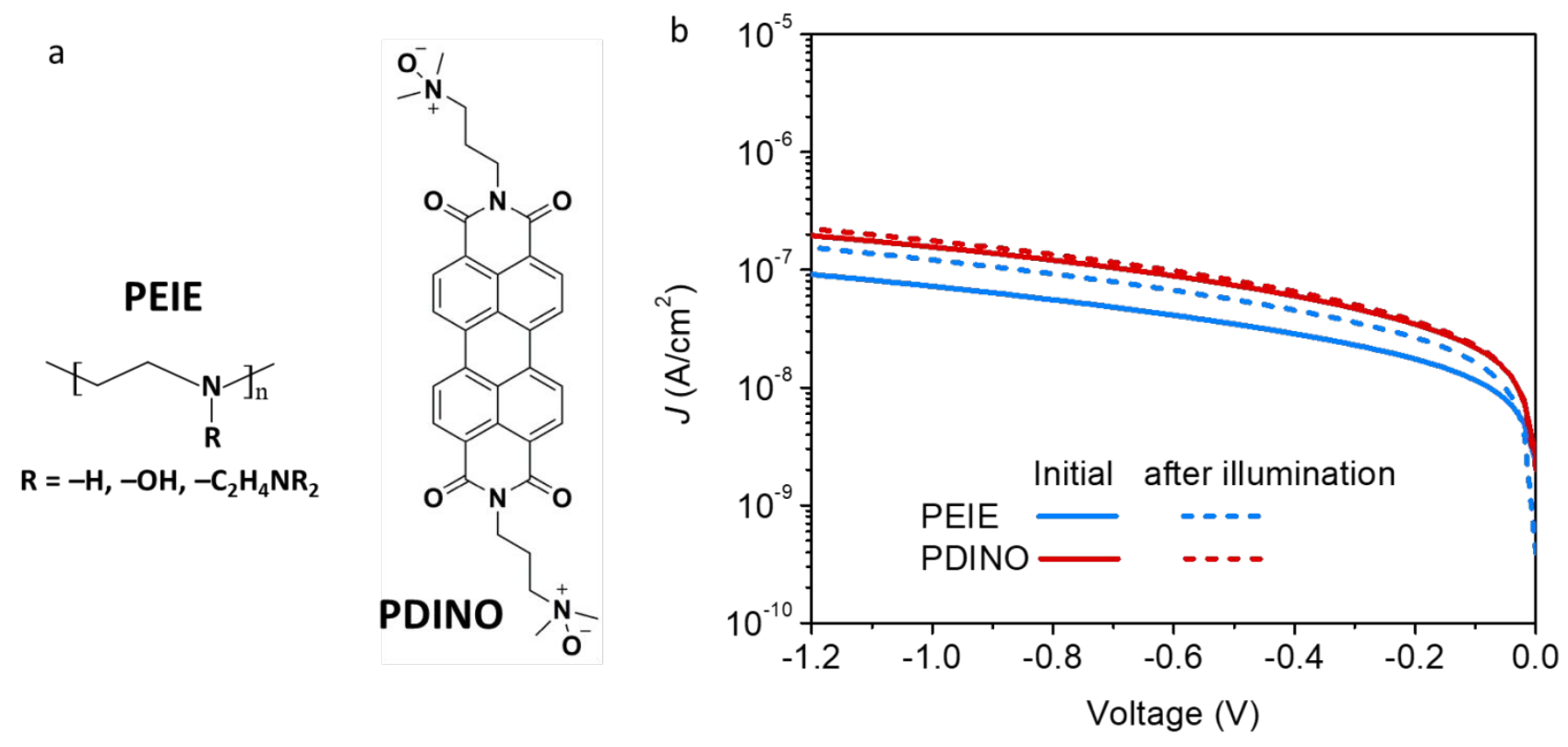

Figure S9. (a) Chemical structures of PEIE and PDINO. (b) Dark $J$ - $V$ characteristics of the OPD devices based on "double" layer PEIE (blue) and PDINO (red) ETLs. 


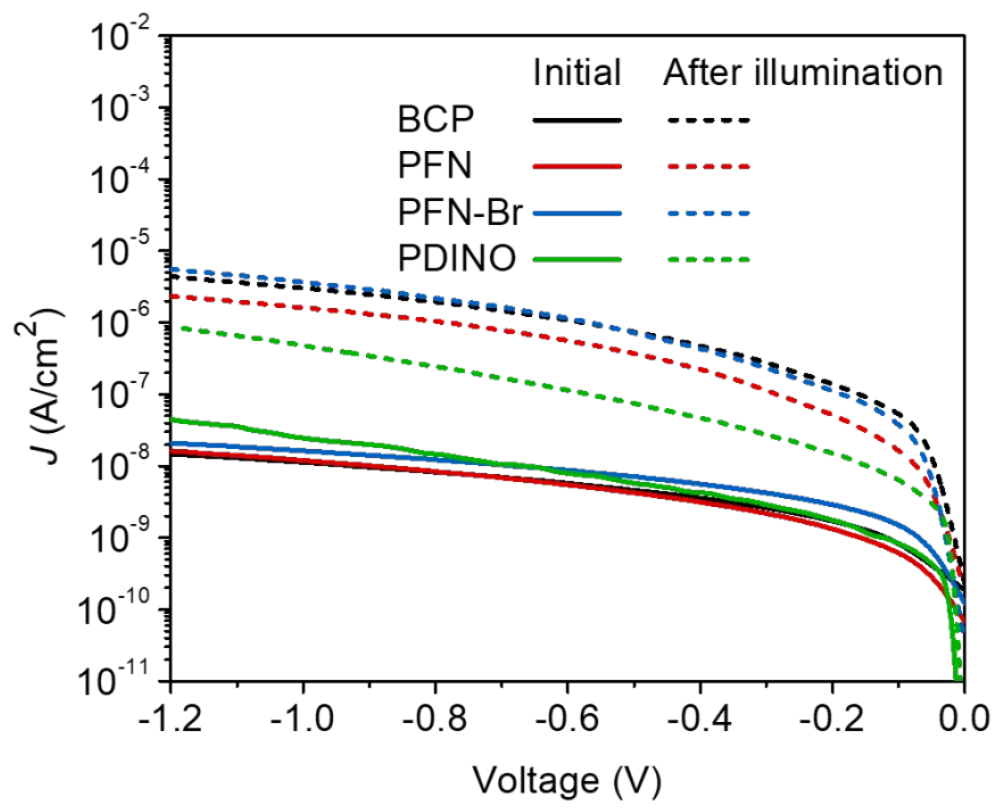

Figure S10. Initial and after-illumination dark $J-V$ characteristics of the inverted devices with different interlayers between the $\mathrm{ZnO}$ ETL and active layer. 

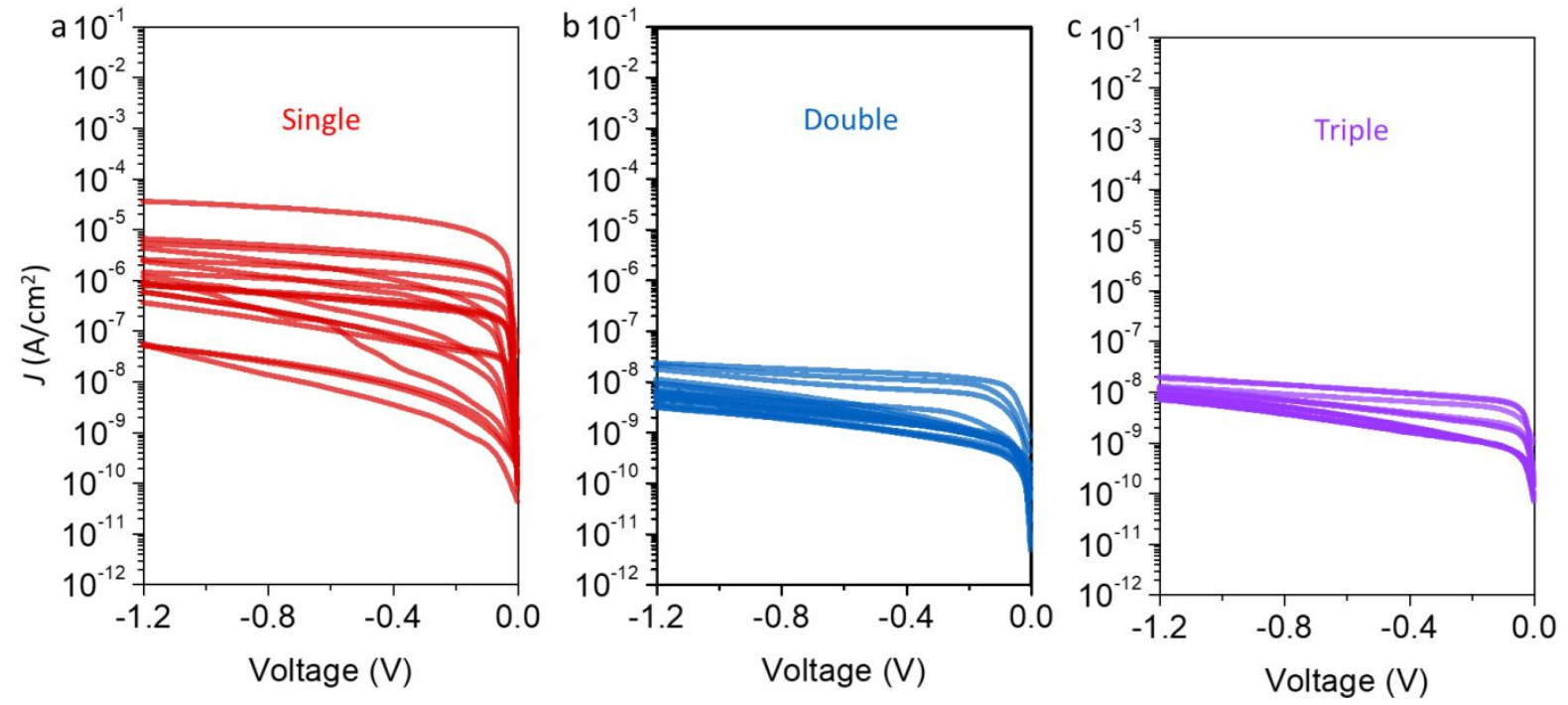

Figure S11. Reverse bias dark $J-V$ curves of the devices based on (a) "single" layer, (b) "double" layer and "triple" layer $\mathrm{SnO}_{2}$ ETLs. Each group has 18 devices. 

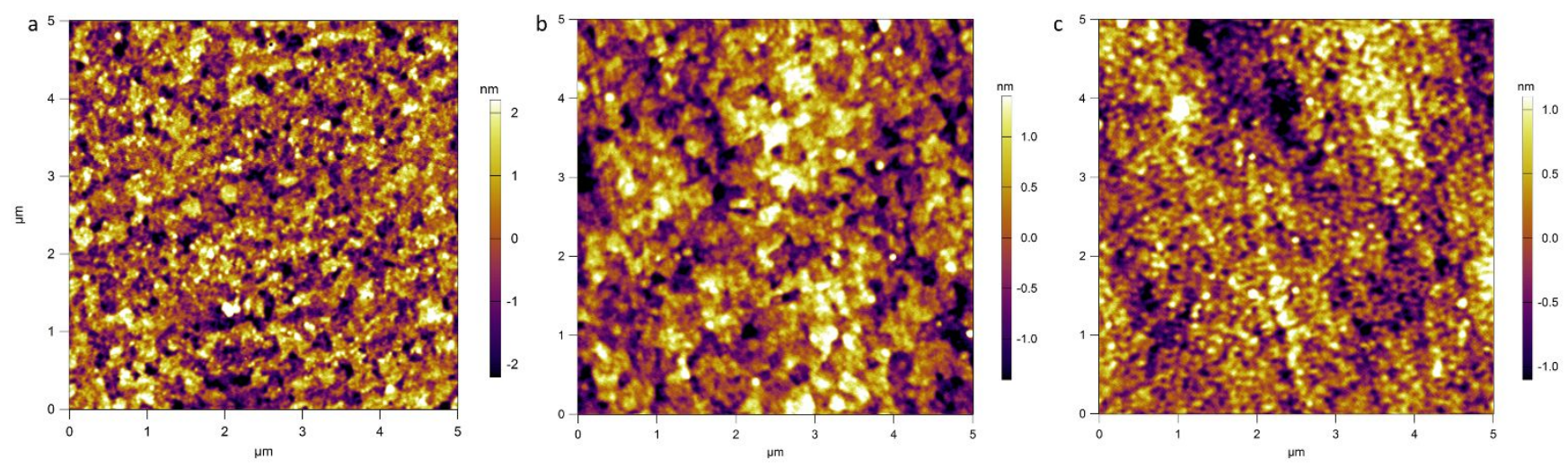

Figure S12. AFM images of (a) single, (b) double and (c) triple layer $\mathrm{SnO}_{2}$ films.

Table S1. Thickness and AFM statistics of the $\mathrm{SnO}_{2}$ films

\begin{tabular}{|c|c|c|c|}
\hline Sample & $\begin{array}{c}\text { Thickness } \\
(\mathbf{n m})\end{array}$ & $\begin{array}{c}\text { Height range } \\
(\mathbf{n m})\end{array}$ & $\begin{array}{c}\text { Height RMS } \\
(\mathbf{n m})\end{array}$ \\
\hline Single layer & 15 & 4.40 & 1.091 \\
\hline Double layer & 24 & 2.80 & 0.699 \\
\hline Triple layer & 34 & 2.20 & 0.546 \\
\hline
\end{tabular}




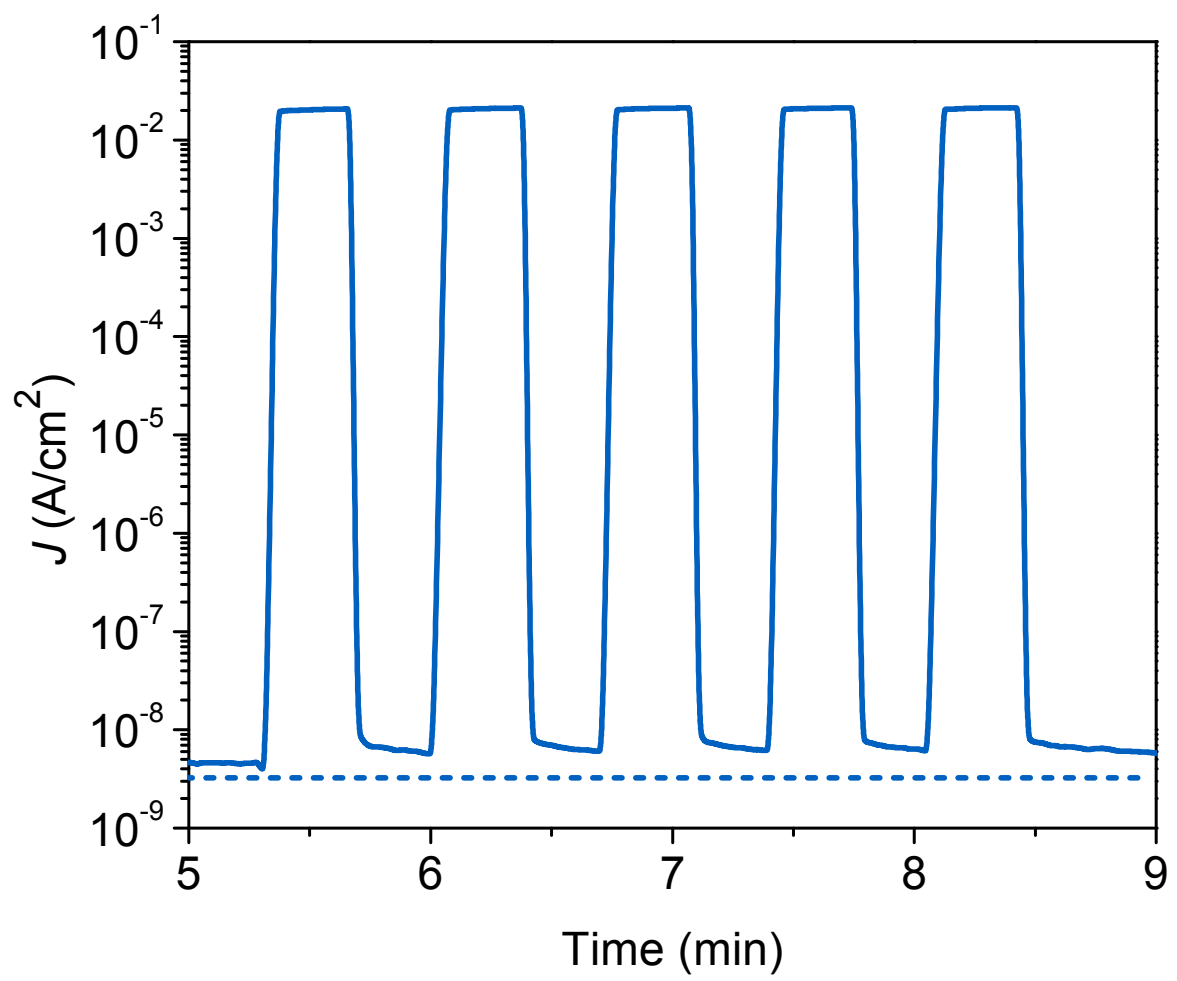

Figure S13. Current response of five consecutive dark-illumination cycles following the 5-min test in Figure $4 \mathrm{c}$. The dashed line indicates the initial dark current prior to any illumination. 


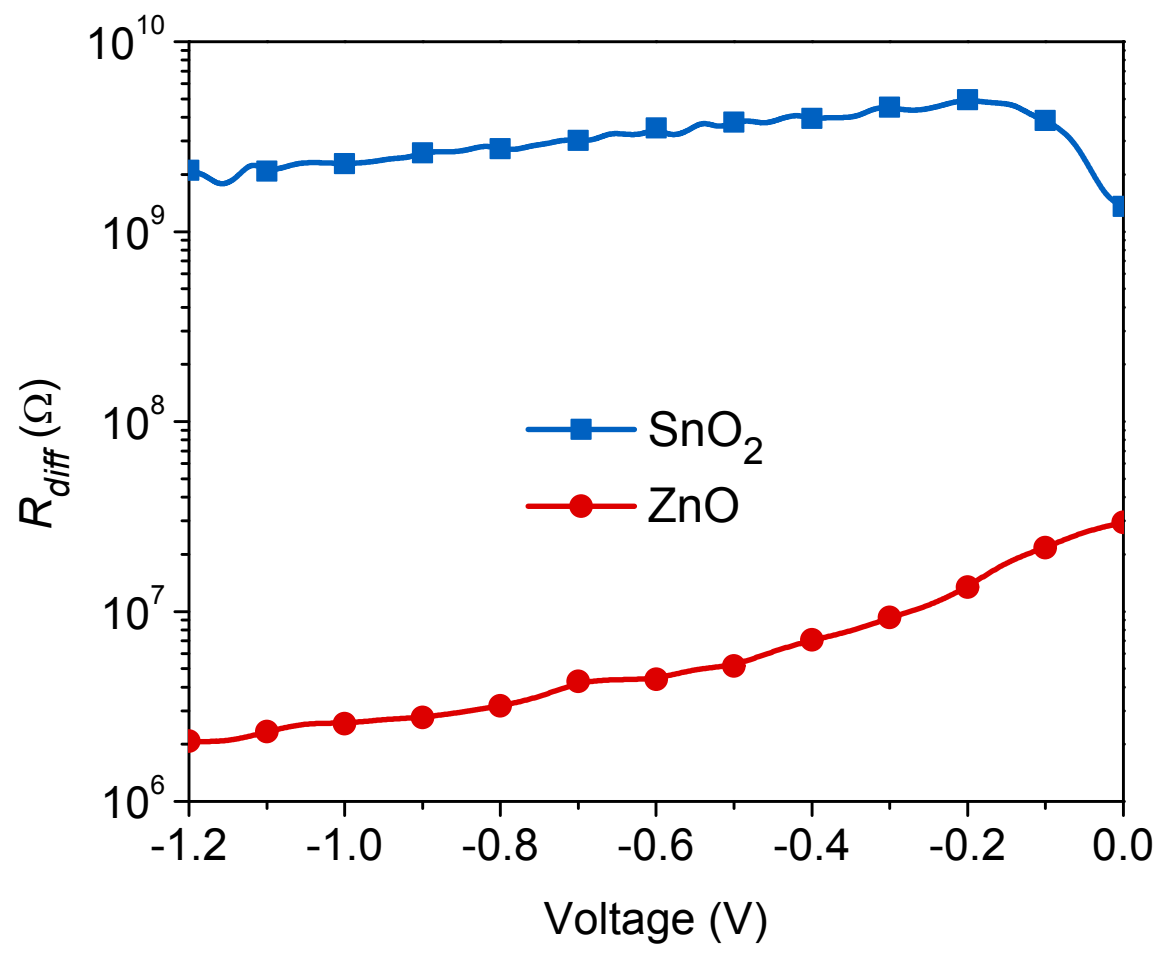

Figure S14. Differential resistance derived from the after-illumination dark current-voltage characteristics of the OPD devices based on $\mathrm{ZnO}$ and $\mathrm{SnO}_{2}$ ETLs. 
a

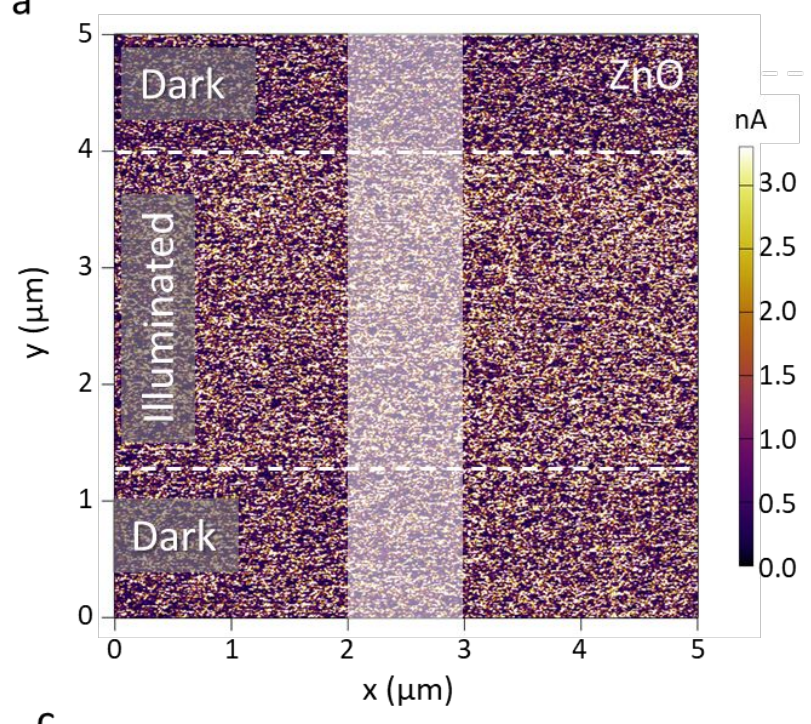

C

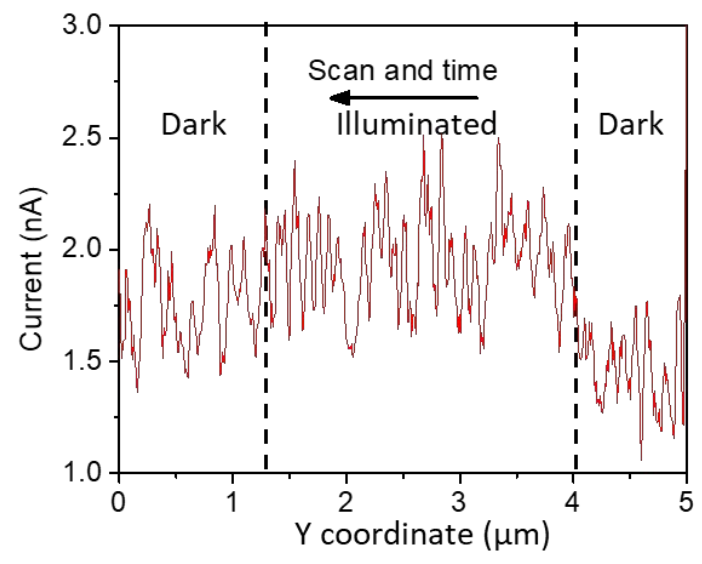

b
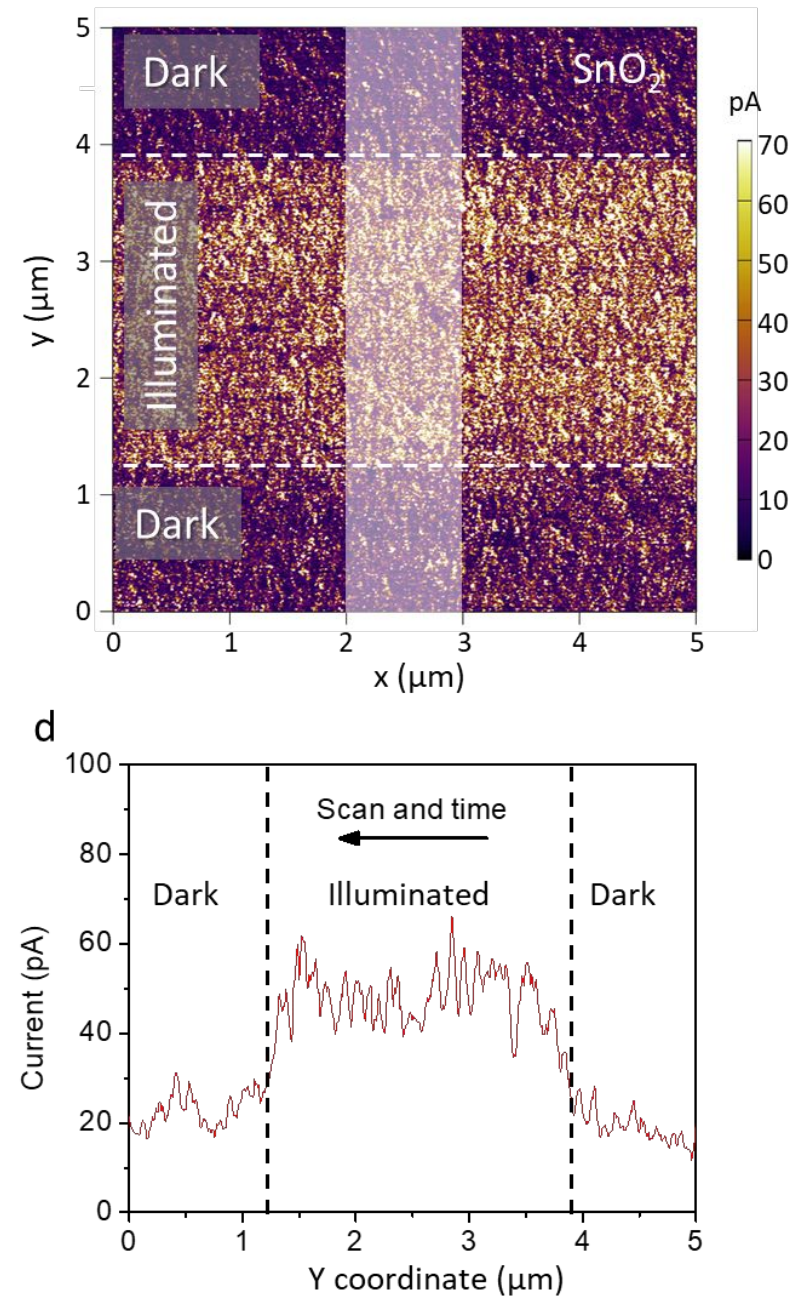

Figure S15. Conductive atomic force microscopy (c-AFM) images collected at $1 \mathrm{~V}$ for (a) $\mathrm{ZnO}$ and (b) $\mathrm{SnO}_{2}$ films. The scan was taken from top (vertical position $5 \mu \mathrm{m}$ ) to bottom (vertical position $0 \mu \mathrm{m}$ ) under first dark, then illuminated, and finally dark conditions. (c-d) Current-vertical coordination characteristics extracted from (a) and (b). The data in (c) and (d) is averaged from current values in the $1 \mu \mathrm{m}$ lateral width indicated by the white area. The black dashed lines in (c) and (d) indicate the illumination conditions during the scan. Please note that the dashed lines indicate only the position of the probe at which illumination was applied or removed. When illumination was applied, the whole areas in Figure S15a and $S 15 b$ were illuminated. 


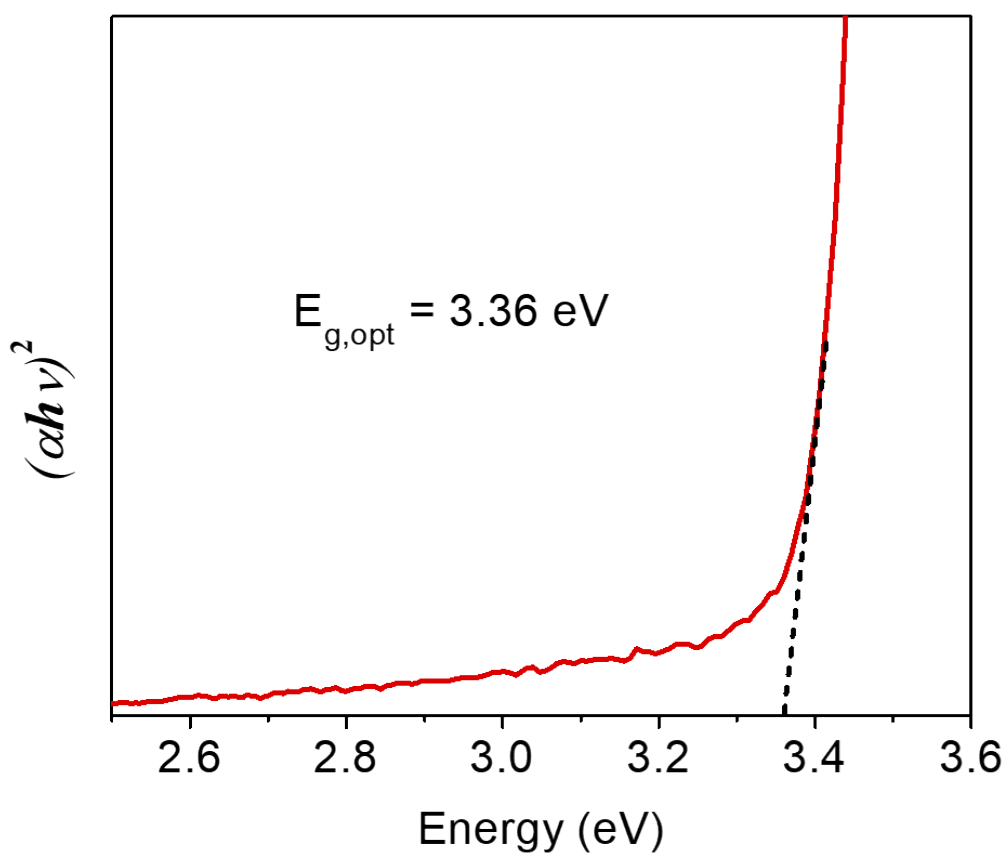

Figure S16. Tauc plot of the $\mathrm{ZnO}$ film. 

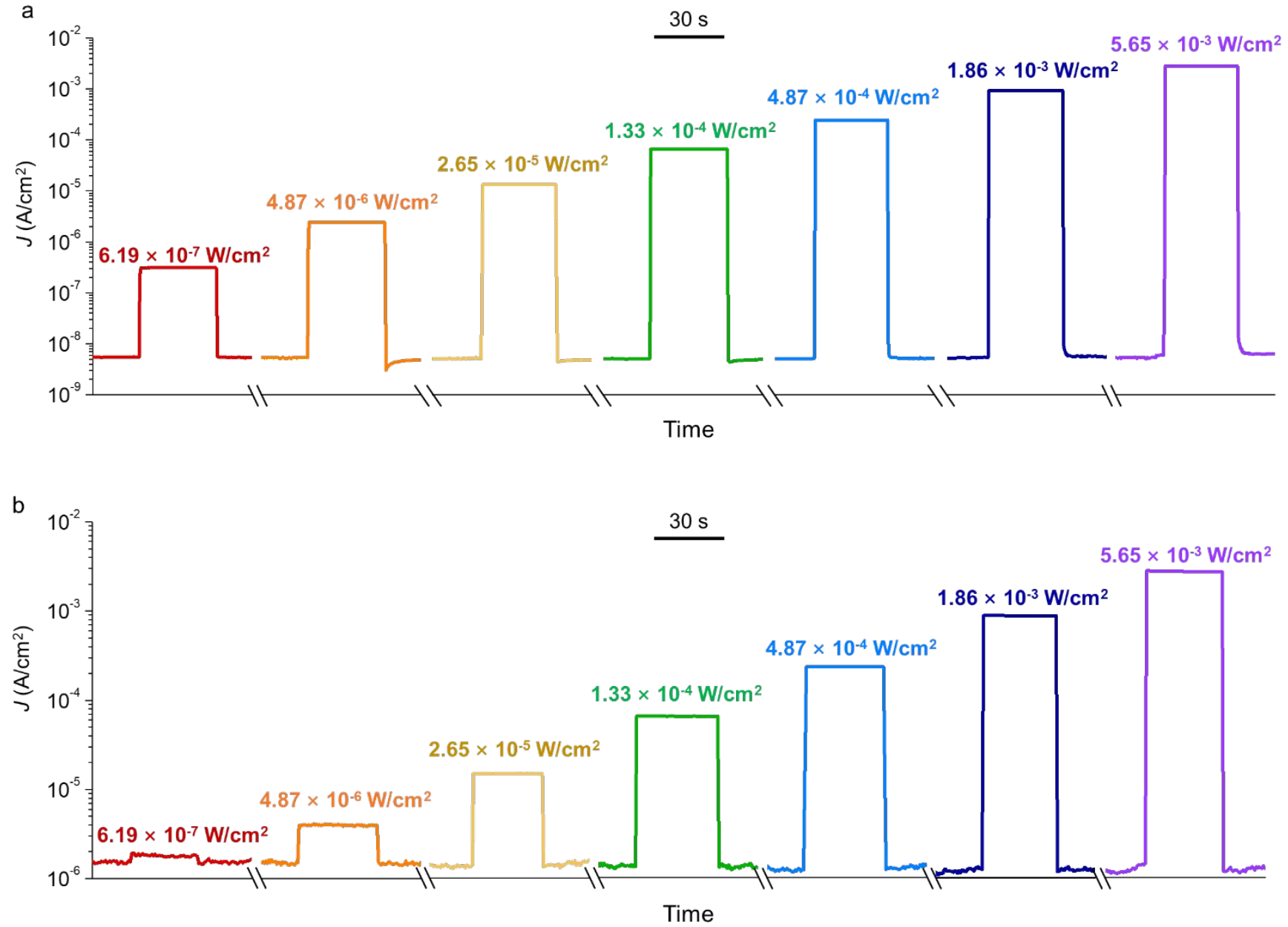

Figure S17. Current response at $-1 \mathrm{~V}$ to different intensities of $940 \mathrm{~nm}$ light for the OPDs based on (a) $\mathrm{SnO}_{2}$ and (b) $\mathrm{ZnO}$ ETLs. 
a
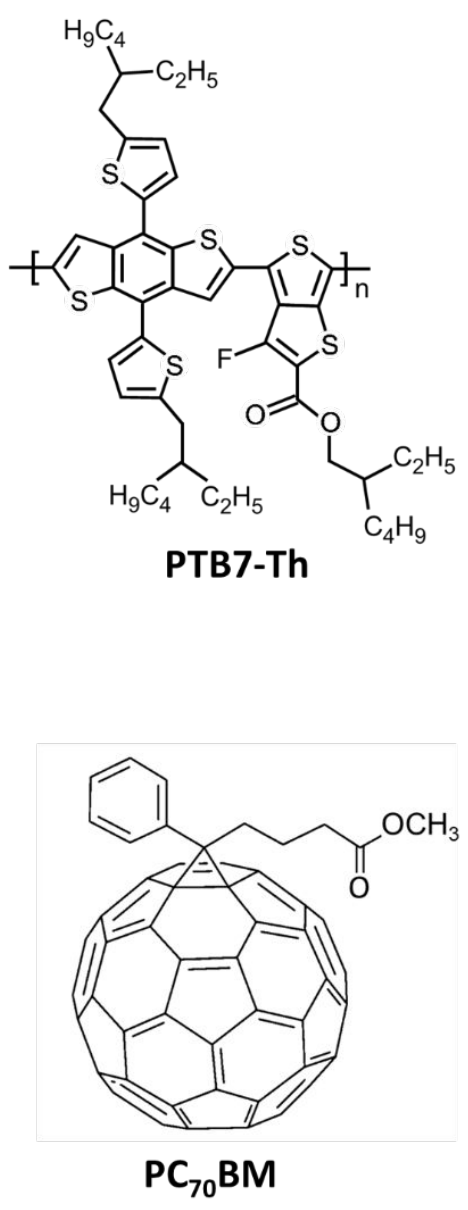
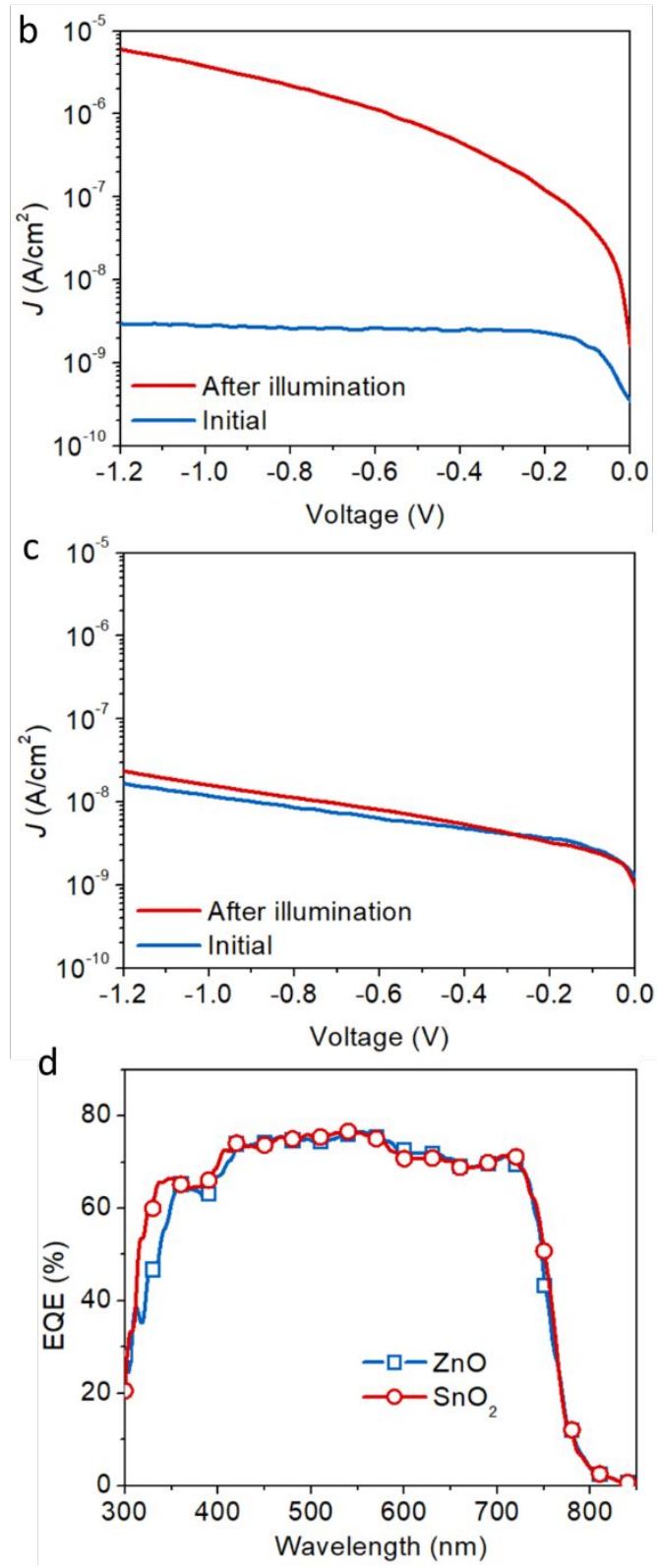

Figure S18. (a) Chemical structures of $\mathrm{PTB} 7-\mathrm{Th}$ and $\mathrm{PC}_{70} \mathrm{BM}$. Initial and after-illumination dark $J-V$ characteristics of the devices with (b) $\mathrm{ZnO}$ and (c) $\mathrm{SnO}_{2}$ as the ETL. (d) EQE profiles of PTB7Th: $\mathrm{PC}_{70} \mathrm{BM}$ OPDs with $\mathrm{ZnO}$ or $\mathrm{SnO}_{2}$ as the ELT at $-1 \mathrm{~V}$. 

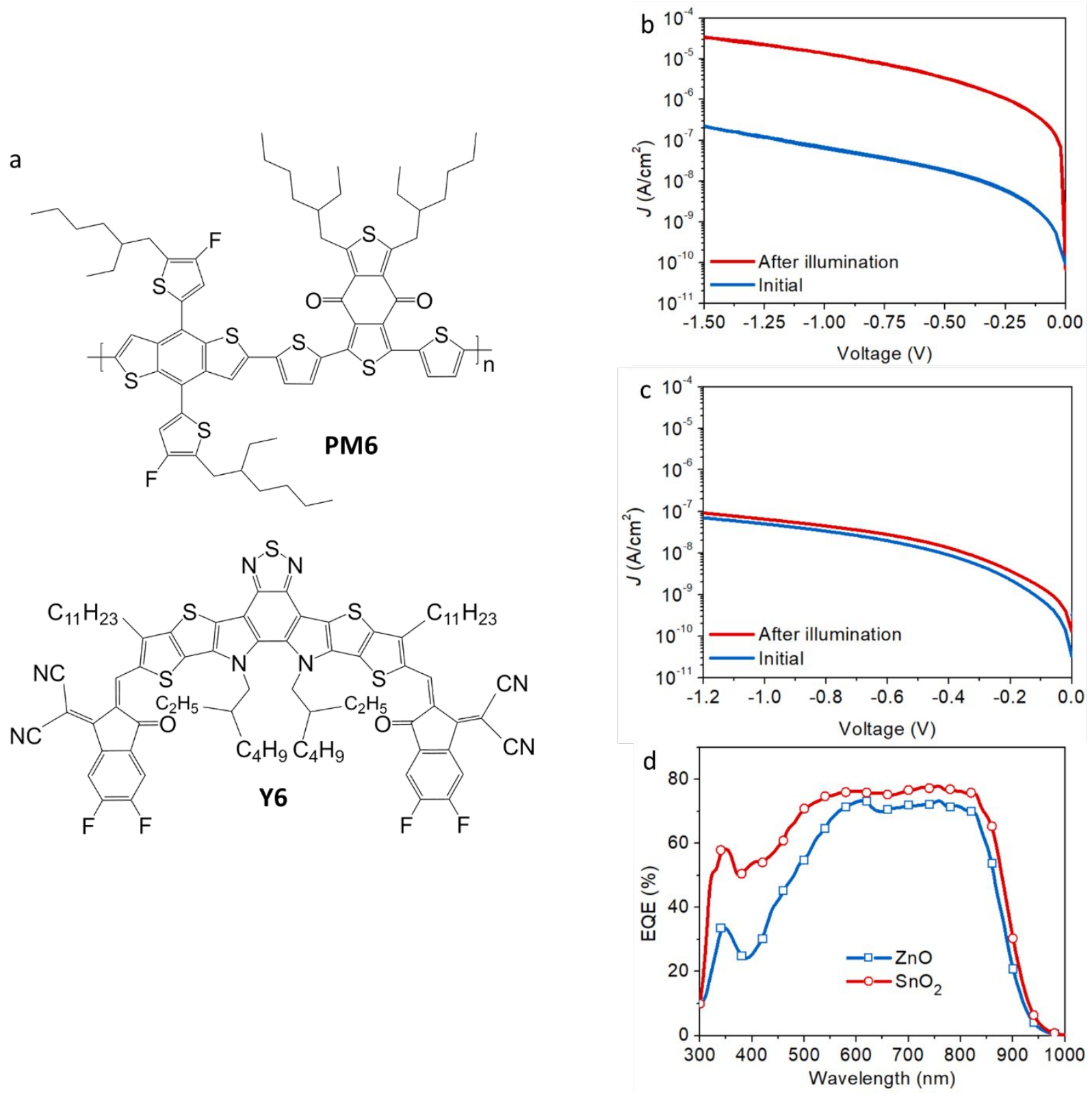

Figure S19. (a) Chemical structures of PM6 and Y6. Initial and after-illumination dark $J-V$ characteristics of the devices with (b) $\mathrm{ZnO}$ and (c) $\mathrm{SnO}_{2}$ as the ETLs. (d) EQE profiles of PM6:Y6 OPDs with ZnO or $\mathrm{SnO}_{2}$ as the ETL at $-1 \mathrm{~V}$. 